Puzzolo Julia ${ }^{1}$

Amarilla Delia Inés ${ }^{2}$

${\text { Colautti Marise }{ }^{3}}^{3}$

Moreno Justina ${ }^{4}$

De Paepe Pierre ${ }^{5}$

Vargas Lorenzo Ingrid 6

Vázquez Navarrete María

Luisa $^{7}$

Maestría en Salud

Pública Centro de

Estudios Interdisciplinarios

Universidad Nacional de

Rosario. Rosario, Argentina.

${ }^{2}$ Maestría en Salud

Pública Centro de Estudios

Interdisciplinarios UNR.

Universidad nacional de

Rosario. Rosario, Argentina.

${ }^{3}$ Maestría en Salud

Pública Centro de

Estudios Interdisciplinarios

Universidad Nacional de

Rosario. Rosario, Argentina.

${ }^{4}$ Maestría en Salud

Pública Centro de

Estudios Interdisciplinarios

Universidad Nacional de

Rosario. Rosario, Argentina.

${ }^{5}$ Prins Leopold Instituut voor

Tropische Geneeskunde.

ITM. Amberes, Bélgica

'Grupo de Investigación

de Políticas de Salud y

Servicios de Salud, Unidad

de Investigación de Políticas de Salud, Consorci de

Salut i Social de Catalunya,

Barcelona, España.

${ }^{7}$ Grupo de Investigación

de Políticas de Salud y

Servicios de Salud, Unidad

de Investigación de Políticas

de Salud, Consorci de

Salut i Social de Catalunya,

Barcelona, España.

Trabajo recibido: 18 de mayo de 2018.

Aprobado: 28 de febrero 2019.

\section{COORDINACIÓN DE} LA ATENCIÓN ENTRE NIVELES Y SUS FACTORES ASOCIADOS EN DOS SUBREDES DE LA RED MUNICIPAL DE SALUD DE LA CIUDAD DE ROSARIO, ARGENTINA.

\author{
COORDINATION OF CARE BETWEEN \\ LEVELS AND THEIR ASSOCIATED \\ FACTORS IN TWO SUBNETS OF THE \\ MUNICIPAL HEALTH NETWORK IN THE CITY \\ OF ROSARIO, \\ ARGENTINA.
}

\section{COORDENAÇÃO DE CUIDADOS ENTRE OS NÍVEIS E FATORES ASSOCIADOS EM DOIS SUB-REDES DA REDE MUNICIPAL DE SAÚDE DA CIDADE DE ROSARIO, ARGENTINA}

\section{Resumen}

Objetivo: evaluar la coordinación de atención entre niveles y factores que influyen a partir de experiencia de médicos de primer y segundo nivel en subredes del sistema público Municipalidad de Rosario.

Método: Estudio transversal, encuestas presenciales a médicos de Primer (AP) y Segundo (AE) nivel. Análisis univariado y bivariado.

Resultados: similares en subredes. Bajo intercambio de información, pero alta valoración. Remisión oportuna entre niveles; prevalece entre AP existencia de acuerdos de indicaciones de médicos de AE. No se repiten estudios. AP es responsable del seguimiento del paciente, AE envía a pacientes al primer nivel post consulta, AE hacen recomendaciones a AP y AP consultan dudas a AE. La minoría percibe atención coordinada. Factores que influyen: edad, nivel de atención, antigüedad 
de trabajo, red de atención, tiempo/paciente, tiempo para coordinación en consulta, satisfacción salarial y confianza en habilidades clínicas. Conclusiones: rasgos comunes con particularidades producto de la construcción de redes locales.

Palabras Clave: Enfermedad crónica - servicios de salud - atención de salud- Argentina

\section{Abstract}

The objective was to evaluate care coordination between levels and influential factors from the experience of Primary Care (PC) and Secondary Care (SC) level doctors in subnets of the public system in the city of Rosario. Methods: Cross sectional study, based on face-to-face surveys to doctors of first and second care levels. Univariate and bivariate analysis. Results: similar in both subnets. Low information exchange, but highly valued. Adequate remission between levels, agreements among PC with SC's recommendations. Studies are not repeated. PC doctor is responsible for the patient's follow up; SC doctor sends patients for a follow up consultation with PC doctor, SC makes recommendations to $\mathrm{PC}$ and $\mathrm{PC}$ asks doubts to SC. A minority perceives coordinated care. Age, care level, seniority at work, care network, time/patient, coordination time in consultation, satisfaction with salary, and confidence on clinical abilities are influential factors. Conclusions: common features with particularities due to the construction of local networks.

Key Words: Chronic disease - Health services - Health care - Argentina.

\section{Resumo}

O objetivo foi avaliar a coordenação do atendimento entre níveis e fatores que influenciam a partir da experiência de médicos de primeiro e segundo nível em sub-redes do sistema público do município de Rosário. O método foi um estudo transversal, enquetes presenciais a médicos do primeiro (AP) e segundo (AE) nível. Análise univariada e bivariada. Resultados: semelhantes em sub-redes. Baixa troca de informações, mas alta valorização. Transmissão oportuna entre os níveis; a existência de acordos de indicações de médicos de EA prevalece entre aqueles de AP. Nenhum estudo é repetido. AP é responsável pelo acompanhamento do paciente. AE envia pacientes para o primeiro nível após consulta, AE faz recomendações para dúvidas de AP, e AP consulta dúvidas para AE. A minoria percebe atenção coordenada. Fatores que influenciam: idade, nível de cuidados, antigüidade no serviço, rede de cuidados, tempo / paciente, tempo de coordenação da consulta, satisfação salarial e confiança nas habilidades clínicas. Conclusões: características comuns com particularidades decorrentes da construção de redes locais.

Palavras chave: Doença crônica - serviços de saúde - cuidados de saúde - Argentina.

\section{Introducción}

Ante la fragmentación de los servicios de salud en América Latina, la Organización Panamericana de la Salud (OPS) considera a la coordinación de la atención como el punto más importante que deben impulsar estos países para mejorar la atención primaria y la integración de los servicios de salud $(1,2)$. En este sentido la evaluación y mejora de la coordinación aparecen como un punto relevante para mejorar la calidad asistencial.

La coordinación entre niveles de atención es definida como la concertación de todos los servicios relacionados con la atención a la salud independientemente del lugar donde se reciben, de manera que se armonicen y se logre un objetivo común sin conflictos; cuando alcanza su grado máximo, la atención se considera integrada (3). Se pueden distinguir tres tipos: de información, gestión clínica y administrativa. La coordinación de la información implica la transferencia y la utilización de información. La coordinación de la gestión 
clínica se define a partir de tres dimensiones: la coherencia de la atención, la accesibilidad entre niveles y el seguimiento adecuado del paciente entre niveles asistenciales. Al mismo tiempo se reconoce que existen tres tipos de factores que influyen: los organizativos, como por ejemplo la existencia de mecanismos de coordinación en la atención, los relacionados a los profesionales que suponen por un lado los valores y condiciones necesarias para tomar decisiones en cuanto a la coordinación $(4,5)$. Finalmente, los relacionados con las características del sistema de salud y su contexto (6). Los estudios que abordan la coordinación de manera integral en América Latina son escasos y en su mayoría solo se centran en algunos de sus aspectos o dimensiones, ellos destacan la limitada coordinación por el deficiente intercambio de información clínica, dificultades en el acceso al segundo nivel y desacuerdos entre profesionales de distintos niveles. Resaltan que las condiciones de trabajo dificultan el uso de los mecanismos de coordinación y no se reconoce al primer nivel como el responsable de coordinar la atención de los pacientes. Aquellos que la trabajan de manera integral concluyen que el nivel de atención, la confianza en las habilidades clínicas del otro profesional, el conocimiento entre los profesionales, el reconocimiento de los médicos de AP como los responsables del seguimiento del paciente, el tiempo disponible para la coordinación en la consulta, la satisfacción con el salario y con el trabajo, influyen en la percepción de Coordinación de la atención (7,8). En Argentina y en Rosario en la literatura existente se ha trabajado cualitativamente y cuantitativamente la experiencia de profesionales y el análisis cuantitativo de registros. Los resultados coinciden a nivel nacional y local en los bajos niveles de contrarreferencia $(9,10,11)$ y la variabilidad del contenido de los mecanismos de información clínica que entorpece el intercambio de la misma $(10,11)$. En Rosario el registro de la información clínica no se encuentra informatizada $(11,12)$. Por otro lado, en esta red existen dispositivos de registros de información que facilitan el seguimiento y la atención integral y longitudinal del paciente pero que sólo pertenecen al primer nivel de atención (11). A diferencia de lo planteado para otras redes de Argentina (9), en la red de Rosario los médicos reconocen que con una articulación entre niveles menos obstaculizada, no habría que recurrir a relaciones personales para obtener una información clínica adecuada. También para Rosario se destaca que el otorgamiento de turnos por sistema de cupos y la distritalización de los especialistas mejoran la organización y acceso al segundo nivel (10). En relación al acceso a estudios de alta complejidad, las posibilidades de acceso adecuado en el tiempo dependen de la especialidad que los prescribe y su relación con la condición de cobertura por obra social (10). En este contexto, los resultados de la línea de base del estudio Equity LA II habilitan a la sistematización y reflexión respecto a la coordinación clínica de la atención en todas sus dimensiones y los factores que la influyen, constituyendo un aporte a la escasa producción sobre el tema en Argentina y Rosario (13)

\section{Caracterización del sistema de salud local}

El sistema de salud argentino, heterogéneo y segmentado, cuenta con tres subsectores: privado, de obras sociales y público (14). El subsector público tiene carácter federal, cada provincia cuenta con potestad para definir funciones, infraestructura y competencias para el área salud. (15) La ciudad de Rosario se encuentra distritalizada en seis áreas y 500.000 personas aproximadamente acuden a efectores de salud estatales. El subsector público de la ciudad está compuesto por efectores que dependen de la jurisdicción provincial y municipal ambos centrados en la estrategia de Atención Primaria de la Salud (APS). La red provincial está compuesta de 28 centros de atención primaria de la salud (CAPS), 1 efector de segundo nivel, 3 hospitales de tercer nivel y 3 maternidades. La municipal cuenta con 52 CAPS, 4 efectores de segundo nivel - 3 hospitales y 1 Centro de Especialidades Médicas Ambulatorias (CEMAR) -, 2 efectores de tercer nivel y dos maternidades. Ambas redes se distribuyen en el mismo territorio y atienden poblaciones de iguales características (16). En el subsector municipal la estrategia de APS se ha implementado 
desde hace más de 20 años y supuso, en cada distrito, un número determinado de CAPS junto a uno o más hospitales de referencia (17). Desde 2003 este trabajo en red se sostiene por el proceso de adscripción de pacientes que implica la conformación de equipos de referencia constituidos por un médico y un enfermero en el primer nivel, responsables de una determinada población a cargo. Esto garantiza el acceso a mayores niveles de complejidad y el seguimiento de los pacientes en el continuo asistencial, proceso que fue acompañado por la descentralización de algunas especialidades desde el segundo y tercer nivel al primero $(18,19)$. El artículo tiene como objetivo evaluar la coordinación de la atención entre niveles y los factores que influyen a partir de la experiencia de los médicos de primer y segundo nivel de atención en dos subredes del sistema de Salud Pública de la Municī̄alidad de Rosario.

\section{Materiales y Métodos}

\section{Diseño y áreas de estudio}

Estudio transversal basado en una encuesta a médicos en dos subredes de la red municipal de Rosario. Esta investigación hizo foco en dos subredes municipales: subred distrito norte/ noroeste $(\mathrm{RN})$ tiene a su cargo la salud de 321227 personas (51,8\% de mujeres y $48,2 \%$ de varones) con 18 CAPS, 2 hospitales de segundo nivel y el CEMAR. La subred distrito sur/ sudoeste (RS) tiene a su cargo a 272075 personas (51,8\% de mujeres y 48,2\% de varones), cuenta con 20 CAPS, 1 hospital de segundo nivel y el CEMAR (20). Ambas subredes se seleccionaron por ser comparables y de acuerdo a los siguientes criterios: a) prestación de un continuo de servicios incluyendo al menos atención primaria y especializada; b) prestación de servicios a una población definida; c) provisión de servicios en áreas urbanas de bajos ingresos; d) disposición a participar e implementar las intervenciones diseñadas; y e) liderazgo con competencia para implementar las estrategias diseñadas.

\section{Población de estudio y muestra}

La población de estudio está compuesta por los médicos/as de primer y segundo nivel que lleven más de tres meses trabajando en la red, que proporcionan atención directa a pacientes y que tienen contacto con el otro nivel de atención (p.ej. a través del proceso de referencia de los pacientes). El tamaño de muestra calculado fue de 174 médicos/as con el fin de asegurar la detección de una variación del 15\% en la percepción de los médicos/ as sobre la coordinación de la atención en las redes, con base a un poder del $80 \%(\beta$ $=0.20) \mathrm{y}$ un nivel de confianza del $95 \%(\beta=0.05)$ en un contraste bilateral. El contacto con los profesionales del primer nivel de ambas subredes, se realizó a través de los jefes de servicios, ante quienes se gestionó la presentación del Proyecto Equity LA II. Se invitó a todo el personal médico y, en aquellos casos con disposición a contestar la encuesta, y que cumplían los criterios de selección, se agendó un encuentro en el lugar de trabajo. El porcentaje de rechazo fue del $8,2 \%$ en RN y $6 \%$ en RS.

\section{Recogida de datos y calidad}

La recogida de datos se realizó de 2015 mediante la aplicación, en entrevistas presenciales, del cuestionario COORDENA AR en su versión 2015 (http://www.equity-la.eu/upload/ seccions/files/COORDENA_AR\%282\%29.pdf). Este instrumento fue adaptado en contenido y lenguaje a la red de Rosario, y posteriormente se sometió a un pretest con 3 entrevistas cognitivas a médicos, seguido de una prueba piloto en la que se aplicaron 20 cuestionarios para evaluar el ritmo y carga de la entrevista, aceptabilidad y comprensión de las preguntas, y colaboración de los entrevistados, entre otros. Como resultado se modificaron o quitaron algunas preguntas para reducir el cuestionario y evitar repetición. La información detallada sobre el diseño y estructura en los seis países ha sido publicada previamente (7). Con el fin de asegurar la calidad de los datos: se realizó una revisión del $100 \%$ de los cuestionarios por parte de los supervisores con retroalimentación al encuestador/a; se realizó una supervisión presencial del 20\% de los cuestionarios (seleccionados aleatoriamente); finalmente se llevó a cabo una doble digitación de todos 
los cuestionarios para minimizar los errores en la carga de datos.

\section{Variables}

Se analizan nueve ítems sobre la experiencia de coordinación clínica como variables de resultados para el análisis descriptivo. Para analizar los factores asociados a la percepción sobre una atención clínica coordinada entre niveles de atención (siempre/ muchas veces) fue utilizada "Creo que la atención entre los médicos de atención primaria y los especialistas en la red está coordinada" dado que actúa como buena medida al estar relacionado a las dimensiones más relevantes de la coordinación de la atención. Se tomaron las siguientes variables explicativas: a) demográficas: sexo y edad; b) condiciones de empleo: nivel de atención, años trabajados en el efector, tipo de contrato, horas semanales de contrato, trabajo complementario en sector privado; c) condiciones organizativas: red, tiempo por paciente, tiempo destinado a la coordinación clínica, el directivo facilita la coordinación entre niveles, supervisión regular de la práctica; d) actitud hacia el trabajo: satisfacción con el trabajo, con el salario y planes de cambio de trabajo en los próximos seis meses; finalmente e) factores relacionales de los profesionales: identificación del médico de atención primaria como el responsable del seguimiento de paciente a través de los niveles de atención, conocimiento de los médicos del otro nivel y confianza en sus habilidades clínicas.

Análisis

Se realiza un análisis univariado de cada una de las variables explicativas por red y análisis bivariado para describir las variables que influyen sobre la coordinación de la atención clínica a través de los factores asociados por red y por nivel de atención. Por lo tanto, se realiza un análisis de regresión logística para probar la posible influencia de los diferentes factores (variables explicativas) sobre la percepción de la coordinación clínica (variable respuesta). Se utilizó un ajuste robusto de la covariancia - empleando la variable red para dar cuenta de las observaciones correlacionadas debido a la agrupación. Se presentan los resultados de la regresión logística a través de las razones de odds de cada uno de los factores con sus respectivos intervalos de confianza (IC 95\%). Dichos factores fueron agregados al modelo manteniendo el siguiente orden: variables demográficas, condiciones de contratación, organización, actitud con respecto al trabajo y factores de interacción entre los médicos de ambos niveles. En el caso en el que ninguna de las variables del grupo sea significativa, al menos una se incluyó en el modelo para determinar el impacto de diferentes tipos de variables en el ajuste del modelo. Se probó, además, la multicolinealidad de las variables explicativas utilizando el factor de inflación de la variancia (VIF), el cual resulto no significativo (con valores menores a 2). Por último, el ajuste del modelo se realizó con el test de bondad de ajuste de Hosmer-Lemenshow. El análisis estadístico se llevó a cabo utilizando el software estadístico (STATA), versión 14.

\section{Consideraciones éticas}

Previo a la entrevista se obtuvo el consentimiento informado de todos los participantes, se les comunicó el derecho a rechazar su participación o retirarse de la encuesta y se les aseguró la privacidad y la confidencialidad de los datos de acuerdo a la legislación vigente. Solo aquellos médicos que aceptaron firmar el consentimiento informado fueron entrevistados. El Consentimiento Informado que se utilizó durante las encuestas fue aprobado por el Comité de Ética de la Secretaría de Salud Municipal y el proyecto de investigación fue inscripto en el registro de protocolos de investigación (Registro provincial $\mathrm{N}^{\mathrm{o}}: 257$ ) del Comité Provincial de Bioética dependiente del Ministerio de Salud y la Secretaría de Estado de Ciencia, Tecnología e Innovación de la Provincia de Santa Fe.

\section{Resultados}

\section{Característica de la muestra:}

La muestra de médicos encuestados consta de un total de 178 médicos en la $\mathrm{RN}$, con 
mayoría de médicos del segundo nivel (110 especialistas de segundo nivel - AE - y 68 médicos de atención primaria - AP -) y en la RS 172 médicos con una distribución más pareja entre niveles. En ambas redes, más de la mitad de la muestra está compuesta por mujeres siendo un poco mayor en la RS. De la misma forma la mayoría de los encuestados tienen entre 36 y 50 años. En la RN y RS, la mayoría de los médicos lleva más de tres años trabajando en el CS u hospital. En el RS, en cambio la mayoría tiene más de diez años de experiencia trabajando en el centro. (Tabla 1)

Tabla Nº1: Características demográficas, laborales y organizacionales de la muestra.

\begin{tabular}{|c|c|c|}
\hline & $\begin{array}{c}\text { RS } \\
(n=172) \\
n(\%)\end{array}$ & $\begin{array}{c}\text { RN } \\
(n=178) \\
n(\%)\end{array}$ \\
\hline \multicolumn{3}{|l|}{ Sexo } \\
\hline Masculino & $44(25,6)$ & $62(34,8)$ \\
\hline Femenino & $128(74,4)$ & $116(65,2)$ \\
\hline \multicolumn{3}{|l|}{ Edad* } \\
\hline 24 a 35 años & $28(16,3)$ & $50(28,1)$ \\
\hline 36 a 50 años & $88(51,2)$ & $72(40,4)$ \\
\hline$>50$ años & $56(32,6)$ & $56(31,5)$ \\
\hline \multicolumn{3}{|l|}{ Nivel de atención** } \\
\hline Primer nivel & $89(51,7)$ & $68(38,2)$ \\
\hline Segundo nivel & $83(48,3)$ & $110(61,8)$ \\
\hline \multicolumn{3}{|c|}{ Tiempo que trabaja en el Centro/ } \\
\hline \multicolumn{3}{|l|}{ Hospital } \\
\hline Menos de 1 año & $20(11,6)$ & $27(15,2)$ \\
\hline Entre 1 y 3 años & $24(14.0)$ & $37(20.8)$ \\
\hline Más de 3 años & $128(74,4)$ & $114(64.0)$ \\
\hline \multicolumn{3}{|l|}{ Tipo de contrato } \\
\hline Planta permanente & $145(84,3)$ & $122(68,5)$ \\
\hline Contrato temporario & $26(15,1)$ & $54(30,3)$ \\
\hline \multicolumn{3}{|c|}{ Horas de trabajo por semana } \\
\hline$<20 \mathrm{hs}$ & $23(13,4)$ & $31(17,4)$ \\
\hline $20-40 \mathrm{hs}$ & $143(83,1)$ & $136(76,4)$ \\
\hline$>40 \mathrm{hs}$ & $6(3,5)$ & $11(6,2)$ \\
\hline \multicolumn{3}{|c|}{ Trabajo adicional en un sector privado } \\
\hline $\mathrm{Si}$ & $61(35,5)$ & $58(32,6)$ \\
\hline No & $111(64,5)$ & $120(67,4)$ \\
\hline \multicolumn{3}{|l|}{ Tiempo por consulta } \\
\hline \multicolumn{3}{|l|}{ Primer nivel } \\
\hline$<15 \min$ & $43(48,3)$ & $25(36,8)$ \\
\hline$>15 \min$ & $46(51,7)$ & $43(63,2)$ \\
\hline \multicolumn{3}{|l|}{ Segundo nivel } \\
\hline$<15 \min$ & $42(50,6)$ & $60(54,5)$ \\
\hline$>15 \mathrm{~min}$ & $30(36,1)$ & $34(30,9)$ \\
\hline \multicolumn{3}{|c|}{$\begin{array}{l}\text { Tiempo de consulta suficiente para } \\
\text { dedicarle a la coordinación clínica }\end{array}$} \\
\hline $\mathrm{Si}$ & $56(32,6)$ & $51(28,7)$ \\
\hline No & $111(64,5)$ & $123(69,1)$ \\
\hline
\end{tabular}

Se agruparon las características en Sí: Siempre y Muchas veces; No: Pocas veces y Nunca.

*p valor $=0.021$

** $\mathrm{p}$ valor $=0.011$ 
Respecto a la intención de cambiar de trabajo, en ambas redes el porcentaje es bajo, siendo mayor entre los médicos AE de la RN, y al contrario en la RS. En ambas redes, los médicos de los dos niveles están satisfechos con su trabajo, sin embargo, menos de la mitad percibe recibir un buen sueldo por el mismo. No obstante, en ambas redes los médicos AP son quienes manifiestan una mayor conformidad salarial. (Tabla 2)

En relación a los factores interaccionales, en la RN y RS los médicos AP se reconocen como responsables del seguimiento de los pacientes a través de los niveles, reconocimiento que también comparten, aunque en menor medida, los médicos AE.

En ambas redes menos de la mitad de los médicos conocen personalmente a sus pares del otro nivel, aunque es levemente superior en la RN. No obstante, confían en las habilidades clínicas de los otros médicos en un alto porcentaje, aunque en menor medida entre los especialistas de la RS. (Tabla 2)

Tabla $\mathbf{N}^{0}$ 2: Factores organizativos de la coordinación

\begin{tabular}{|c|c|c|}
\hline & $\begin{array}{c}\text { RS } \\
(n=172) \\
n(\%)\end{array}$ & $\begin{array}{c}\text { RN } \\
(n=178) \\
n(\%)\end{array}$ \\
\hline \multicolumn{3}{|c|}{ Satisfacción con el trabajo } \\
\hline Primer nivel & $67(75,3)$ & $50(73,5)$ \\
\hline Segundo nivel & $60(72,3)$ & $85(77,2)$ \\
\hline Total & $127(73,8)$ & $135(75,9)$ \\
\hline \multicolumn{3}{|c|}{ Planes de cambiar de trabajo en los próximos 6 meses } \\
\hline Primer nivel & $7(7,9)$ & $4(5,9)$ \\
\hline Segundo nivel & $4(4,8)$ & $17(15,5)$ \\
\hline Total & $11(6,4)$ & $21(11,8)$ \\
\hline \multicolumn{3}{|c|}{ Satisfacción con el salario* } \\
\hline Primer nivel & $38(42,7)$ & $27(39,7)$ \\
\hline Segundo nivel & $22(26,5)$ & $31(28,1)$ \\
\hline Total & $60(34,9)$ & $58(32,6)$ \\
\hline \multicolumn{3}{|c|}{$\begin{array}{l}\text { Los médicos de AP son los responsables } \\
\text { del seguimiento de los pacientes en su trayectoria } \\
\text { por los distintos niveles* }\end{array}$} \\
\hline Primer nivel & $77(86,5)$ & $57(83,8)$ \\
\hline Segundo nivel & $54(65,0)$ & $67(60,9)$ \\
\hline Total & $131(76,1)$ & $124(69,7)$ \\
\hline \multicolumn{3}{|c|}{ Conozco personalmente a los médicos del otro nivel* } \\
\hline Primer nivel & $27(30,4)$ & $22(32,3)$ \\
\hline Segundo nivel & $27(32,5)$ & $38(34,6)$ \\
\hline Total & $54(31,4)$ & $60(33,7)$ \\
\hline \multicolumn{3}{|c|}{$\begin{array}{l}\text { Confío en las habilidades clínicas de los médicos de } \\
\text { otros niveles* }\end{array}$} \\
\hline Primer nivel & $73(82,0)$ & $54(79,4)$ \\
\hline Segundo nivel & $57(68,7)$ & $82(74,6)$ \\
\hline Total & $130(75,6)$ & $136(76,4)$ \\
\hline
\end{tabular}

Se agruparon las características en Sí: Siempre y Muchas veces; No: Pocas veces y Nunca. Aquí sólo se muestran la categoría sí.

Experiencia de coordinación de la información y coordinación de la gestión clínica: Respecto al intercambio de información, en ambas redes, es bajo el porcentaje de médicos que reportan que se intercambia información frecuentemente, siendo en la RS levemente inferior. Ahora bien, si se observa la distribución entre niveles, en ambas redes el intercambio de información es mayor entre la AE que entre AP. En las dos redes, aunque 
con mayor porcentaje en la RS, la mayoría de los médicos manifiestan que la información intercambiada es la necesaria para la atención de los pacientes (Tabla 3).

Tabla $\mathbf{N}^{0}$ 3: Experiencia de intercambio de la información

\begin{tabular}{|c|c|c|}
\hline & $\begin{array}{c}\text { RS } \\
(\mathbf{n}=172) \\
\mathbf{n}(\%)\end{array}$ & $\begin{array}{c}\text { RN } \\
(\mathbf{n}=178) \\
\mathbf{n}(\%)\end{array}$ \\
\hline \multicolumn{3}{|c|}{$\begin{array}{l}\text { Médicos de AP y AE intercambia información clínica } \\
\text { sobre la atención (diagnósticos, estudios, } \\
\text { tratamientos) de los pacientes* }\end{array}$} \\
\hline Médicos AP & $28(31,5)$ & $25(36,8)$ \\
\hline Médicos AE & $31(37,3)$ & $45(40,9)$ \\
\hline Total & $59(34,3)$ & $70(39,3)$ \\
\hline \multicolumn{3}{|c|}{$\begin{array}{l}\text { La información intercambiada es la necesaria para la } \\
\text { atención de los pacientes* }\end{array}$} \\
\hline Médicos AP & $66(74,2)$ & $49(72,1)$ \\
\hline Médicos AE & $60(72,3)$ & $78(69,1)$ \\
\hline Total & $126(73,3)$ & $125(70,2)$ \\
\hline \multicolumn{3}{|c|}{$\begin{array}{l}\text { Médicos de AP y AE tenemos en cuenta la } \\
\text { información queIntercambiamos de los pacientes* }\end{array}$} \\
\hline Médicos AP & $63(70,8)$ & $46(67,6)$ \\
\hline Médicos AE & $64(77,1)$ & $84(76,4)$ \\
\hline Total & $127(73,8)$ & $130(73,0)$ \\
\hline
\end{tabular}

Los resultados corresponden a las categorías siempre y muchas veces

Relacionado a la experiencia de coordinación de la gestión clínica, existe una alta consistencia de la atención, principalmente entre los médicos AP de ambas redes. Respecto a la no repetición de los estudios y la inexistencia de contraindicaciones y duplicaciones en los tratamientos, en ambas redes los porcentajes son altos. Sin embargo, el porcentaje de médicos de AE que reportan acuerdo en tratamientos o adecuación en las derivaciones de los médicos de AP es baja, sobre todo en la RS.

En relación al seguimiento de los pacientes, en ambas redes, la mayoría de los médicos refieren que los médicos $\mathrm{AE}$ envían a los pacientes al primer nivel para su seguimiento, aunque el porcentaje es mayor en la RS. No obstante, en ambas redes, menos de la mitad de los médicos de AP refieren recibir recomendaciones por parte de los especialistas, contrastando con los médicos de AE que en más de la mitad de los casos refieren hacerlo. En ambas redes, cerca de la mitad de los médicos de los dos niveles indican que los médicos AP consultan dudas a los especialistas (Tabla 4) 
Tabla $\mathbf{N}^{0}$ 4: Experiencia de Coordinación de la gestión clínica: Coherencia de la atención; Seguimiento Adecuado del paciente; Accesibilidad entre niveles

\begin{tabular}{ccc} 
RS & RN \\
$(\mathbf{n}=172)$ & $(\mathbf{n}=178)$ \\
$\mathbf{n}(\%)$ & $\mathbf{n}(\%)$ \\
\hline
\end{tabular}

Consistencia de la atención entre niveles*

Los médicos no repetimos los estudios que previamente

han realizado los médicos de otro nivel

$\begin{array}{lll}\text { Médicos AP } & 85(95,5) & 60(88,2) \\ \text { Médicos AE } & 66(79,5) & 88(79,9) \\ \text { Total } & 151(87,9) & 148(85,9)\end{array}$

Los médicos estamos de acuerdo con los tratamiento que los médicos de otro nivel han prescrito

Médicos AP

$65(73,1) \quad 48(70,5)$

Médicos AE

$34(40,9) \quad 43(39,1)$

Total

$99(57,7) \quad 91(51,1)$

No existen contraindicaciones y/o duplicaciones en

los tratamientos que los médicos de AP y los de AE

prescribimos

Médicos AP

$76(85,3) \quad 56(82,4)$

Médicos AE

$71(85,5) \quad 84(76,4)$

Total

$147(85,4) \quad 140(78,6)$

Los médicos de AP derivan los pacientes a los $\mathrm{AE}$

cuando es necesario

Médicos AP

$80(89,9) \quad 63(92,6)$

Médicos AE

$41(49,4) \quad 67(60,9)$

Total

Seguimiento del paciente*

$121(70,3) \quad 130(73,0)$

Después de ser atendido por el especialista, el paciente realiza una consulta de seguimiento con el médico de atención primaria

Médicos AP

Médicos AE

Total

Los especialistas hacen recomendaciones(diagnóstico,

tratamiento) al médico de AP sobre el seguimiento de los pacientes

Médicos AP

Médicos AE

Total

$71(79,8) \quad 58(85,3)$

$55(66,3) \quad 67(61,0)$

$126(73,2) \quad 125(70,2)$

Los médicos de AP consultan a los especialistas las dudas que tienen sobre el seguimiento de los pacientes

Médicos AP

Médicos AE

Total

Accesibilidad entre niveles*

$\begin{array}{ll}28(31,5) & 24(35,3) \\ 58(69,8 & 69(62,7) \\ 86(50,0) & 93(52,2)\end{array}$

Al ser derivado al especialista, el paciente espera

mucho tiempo hasta el día de la consulta

$\begin{array}{lll}\text { Médicos AP } & 65(73,0) & 54(79,4) \\ \text { Médicos AE } & 54(65,1) & 64(58,2) \\ \text { Total } & 119(69,1) & 118(66,3)\end{array}$

Tras la consulta con el especialista, cuando el paciente

$46(51,7) \quad 33(48,5)$

$38(45,8 \quad 57(51,8)$

$84(48,9) \quad 90(50,6)$

solicita una consulta con el médico de AP espera

mucho tiempo hasta el día de la consulta

\begin{tabular}{lll} 
Médicos AP & $6(6,7)$ & $7(10,3)$ \\
Médicos AE & $19(22,9)$ & $22(20,0)$ \\
Total & $25(14,6$ & $29(16,3)$ \\
\hline
\end{tabular}

Se agruparon las características en Sí: Siempre y Muchas veces; No: Pocas veces y Nunca.

Aquí sólo se muestran la categoría sí. 
Finalmente, en relación a los tiempos de espera para la consulta con el otro nivel, la mayoría de los médicos en ambas redes, aunque especialmente los médicos de AP, considera que los pacientes deben esperar mucho tiempo para la consulta con el especialista tras la derivación. La percepción de tiempo de espera para ser atendido con el médico de AP tras consultar a la AE, es mejor, sobre todo por parte de los médicos de AP.

Percepción general de buena coordinación entre niveles y los factores asociados:

Para el análisis de los factores asociados con la percepción de coordinación entre niveles, se partió de una afirmación: "creo que la atención entre los médicos de atención primaria y los especialistas en la red está coordinada". En ambas redes, se percibe una baja percepción de la coordinación entre los médicos, similar entre niveles asistenciales, aunque en la RS es apenas más alta (Tabla 5)

Tabla N 5: Percepción general de la Coordinación de la Atención

\begin{tabular}{lcc} 
& $\begin{array}{c}\mathbf{R S} \\
(\mathbf{n}=\mathbf{1 7 2}) \\
\mathbf{n}(\mathbf{\%})\end{array}$ & $\begin{array}{c}\mathbf{R N} \\
(\mathbf{n}=\mathbf{1 7 8}) \\
\mathbf{n}(\mathbf{\%})\end{array}$ \\
\hline $\begin{array}{l}\text { Creo que la atención entre los médicos de atención primaria y especializada está } \\
\text { coordinada* }\end{array}$ & \\
Médicos AP & $20(22,5)$ & $14(20,6)$ \\
Médicos AE & $22(26,5)$ & $27(24,6)$ \\
Total & $42(24,4)$ & $41(23,0)$ \\
\hline
\end{tabular}

Se agruparon las características en Sí: Siempre y Muchas veces; No: Pocas veces y Nunca.

Aquí sólo se muestran la categoría sí.

Varios factores entre las diferentes variables explicativas estudiadas se asocian positivamente a una mejor percepción de la coordinación: edad, nivel de atención, tiempo de trabajo en el centro, red de atención, tiempo por paciente, tiempo dedicado a la coordinación clínica en la consulta, satisfacción con el salario y la confianza en las habilidades clínicas del médico del otro nivel. (Tabla 6) 
Tabla N6: Factores asociados con la alta percepción de Coordinación de la Atención

\begin{tabular}{|c|c|c|}
\hline & $\begin{array}{l}\text { Razón de } \\
\text { odds }\end{array}$ & IC $(95 \%)$ \\
\hline \multicolumn{3}{|l|}{ Sexo } \\
\hline Masculino & 1 & 1 \\
\hline Femenino & 0,768 & $(0,552-1,070)$ \\
\hline \multicolumn{3}{|l|}{ Edad } \\
\hline $24-35$ años & 1 & 1 \\
\hline $36-50$ años & 1,939 & $(1,669-2,254)$ \\
\hline$>50$ años & 2,509 & $(1,482-4,246)$ \\
\hline \multicolumn{3}{|l|}{ Nivel de atención } \\
\hline Primer nivel & 1 & 1 \\
\hline Segundo nivel & 1,327 & $(1,211-1,458)$ \\
\hline \multicolumn{3}{|l|}{ Tipo de contrato } \\
\hline Planta permanente & 0,478 & $(0,157-1,455)$ \\
\hline \multicolumn{3}{|l|}{ Contrato temporario } \\
\hline \multicolumn{3}{|c|}{ Tiempo que trabaja en el Centro } \\
\hline Menos de 1 año & 1 & 1 \\
\hline Entre 1 y 3 años & 0,716 & $(0,261-1,965)$ \\
\hline Más de 3 años & 1,138 & $(1,057-1,224)$ \\
\hline \multicolumn{3}{|l|}{ Red } \\
\hline Sur/Suroeste & 1 & 1 \\
\hline Noroeste/Norte & 1,058 & $(1,011-1,108)$ \\
\hline \multicolumn{3}{|l|}{ Tiempo por paciente } \\
\hline$<15$ min & 1 & 1 \\
\hline$>15 \mathrm{~min}$ & 0,583 & $(0,460-0,738)$ \\
\hline \multicolumn{3}{|c|}{ Horas de trabajo por semana } \\
\hline$<20 \mathrm{hs}$ & 1,892 & $(0,340-10,539)$ \\
\hline $20-40 \mathrm{hs}$ & 2,060 & $(0,415-10,222)$ \\
\hline
\end{tabular}

$>40 \mathrm{hs}$

Tiempo de consulta suficiente para dedicarle a la coordinación clínica

No

$\mathrm{Si}$

Satisfacción con el salario

No

$\mathrm{Si}$

Satisfacción con el trabajo

$\mathrm{Si}$

No

$2,060 \quad(0,415-10,222)$

Identifica a los médicos de AP como responsables del seguimiento del paciente

No

$\mathrm{Si}$

Conocimiento entre profesionales

No

$\begin{array}{cc}1 & 1 \\ \mathbf{1 , 1 5 8} & (\mathbf{1 , 0 9 1}-\mathbf{1 , 2 2 7}) \\ 1 & 1 \\ \mathbf{1 , 7 6 3} & (\mathbf{1 , 3 5 9}-\mathbf{2 , 2 8 7 )} \\ 1,525 & (0,752-3,094)\end{array}$

$\mathrm{Si}$

Confianza en las habilidades clínicas del otro profesional

No

$\begin{array}{cc}1 & 1 \\ 1,659 & (0,784-3,509)\end{array}$

$\mathrm{Si}$ 
La coordinación en todas sus dimensiones es un tema escasamente trabajado en Argentina, y particularmente en Rosario, esta investigación constituye un valioso aporte para la indagación sobre la coordinación de la atención clínica. Se basa en una encuesta a médicos y utiliza una herramienta común para medir de forma integral su experiencia en relación a la coordinación como fenómeno multidimensional y para explorar factores individuales y organizacionales que pueden contribuir a ello (8).

Los resultados de las subredes analizadas no presentan diferencias estadísticamente significativas, excepto por el sexo y la edad de los médicos entrevistados. La similitud en los resultados para el resto de las variables entre las subredes se debe a su pertenencia a una misma red de salud, ambas dependientes de la órbita municipal y en consecuencia funcionando bajo los mismos lineamientos organizacionales.

No obstante, los resultados obtenidos, concuerdan con los resultados encontrados en estudios internacionales del mismo equipo de investigación (2, 6- 8.)

Se observa, al igual que en otros países de América Latina, que la percepción general de coordinación es baja (7). Sin embargo, y en concordancia con los artículos comparativos, al analizar los resultados para las distintas dimensiones de la coordinación se encuentra que para el caso de Rosario hay deficiente intercambio de información, pero mejores experiencias de consistencia y coherencia de la atención, seguimiento del paciente y accesibilidad.

En cuanto al intercambio de información clínica si bien los porcentajes son bajos se considera que la información intercambiada es la necesaria y es tenida en cuenta por los médicos que refieren intercambiarla. El bajo porcentaje de este intercambio tendría que ver con aspectos relacionados a los profesionales que no utilizan o no completan en su totalidad la hoja de referencia y contrarreferencia o se extravía cuando el paciente va de un nivel al otro, este resultado coincide con los encontrados por otros trabajos a nivel local $(10,11)$ y con los hallazgos de otros países $(6)$. Las fallas en la comunicación son consideradas como una de las causas principales de baja coordinación por la literatura sobre el tema, al mismo tiempo que también dan cuenta de estas deficiencias, la falta de información clínica clave al momento de la transferencia de información clínica (21). Como muestran los resultados la alta valoración de los médicos sobre la información intercambiada, si bien escasa, sería un punto de referencia para pensar si las debilidades en este sentido se encuentran en características individuales de los profesionales o en el soporte y características de los mecanismos de información.

Se percibe una buena consistencia de la atención, la mayoría de los médicos refiere no repetir estudios ni tampoco duplicaciones o contradicciones en los tratamientos prescriptos. En este sentido se pueden destacar dos características organizativas del sistema de salud local que fundamentan estos resultados: en primer lugar, la existencia de un laboratorio de análisis bioquímicos central que concentra todas las muestras de estudios de baja, mediana y alta complejidad proveniente de los tres niveles de atención con acceso a través del sistema informático a todos los exámenes complementarios realizados por un paciente en la red. En segundo término, el funcionamiento de áreas de auditoría de procesos de atención que trabajan en efectores hospitalarios y en la Dirección de Centros de Salud que dependen de Auditoría Central, donde se analizan las solicitudes de procedimientos específicos impidiendo su duplicación.

En cuanto al seguimiento de pacientes los médicos de AE dicen hacer recomendaciones al médico de AP para realizarlo, sin embargo, dicha percepción no es compartida por los médicos de AP. Este resultado podría explicarse por el bajo porcentaje de médicos que refieren conocer personalmente a su par del otro nivel, sobre todo teniendo en cuenta la importancia de la colaboración mutua y el conocimiento como un elemento fundamental para construir y sostener redes de salud y su influencia en la utilización de mecanismos 
de coordinación $(9,22)$. No obstante, estos niveles de conocimiento personal entre los médicos de AP y AE, en relación a los resultados obtenidos en otros países de América Latina, son más elevados en coincidencia con los hallazgos comunicados por Vargas 2017 para Uruguay y en menor medida en Argentina. La explicación puede encontrarse en las características de la organización de la red de salud de Rosario donde los médicos de AP forman parte de equipos que son los responsables de la adscripción de pacientes, cuentan con un rol definido y reconocido en la red de salud. En ese sentido la figura del médico de AP es reconocida de manera mayoritaria por todos los médicos de ambas redes como responsable del seguimiento del paciente en su continuo de atención $(10,19,23)$.

Respecto a los factores asociados a la percepción de coordinación se observa, entre los factores organizacionales, que quienes tienden a percibir una atención más coordinada son los médicos con más de 36 años, que llevan más de tres años trabajando en el mismo centro, tienen más de 15 minutos para atención de los pacientes y refieren tener tiempo dentro de la consulta para dedicar a la coordinación clínica. También encontramos que quienes se sienten conformes con el salario que reciben, perciben mayores niveles de coordinación que aquellos que no, en este sentido es claro que en general todas las personas que están conformes y satisfechas con sus condiciones laborales estarán más dispuestas para trabajar en lo que la organización requiere en coincidencia con lo encontrado por Vargas para otros países de la región (7).

Otro factor de influencia es el nivel de atención, los médicos de segundo nivel perciben una atención coordinada más frecuentemente que los de primer nivel, este resultado concuerda con trabajos internacionales que plantean que el mismo fenómeno es percibido de manera diferente por los médicos de los distintos niveles $(6,8)$. En el caso de la red de Rosario, el estudio cualitativo de este proyecto hecha luz sobre la sensación de sobredemanda en la atención y la creciente complejidad de los casos que deben atender los médicos de APS y que pueden ser un aporte para entender dichas diferencias.

Los médicos de la subred de RN también tienen una mejor percepción de coordinación. Este constituye un punto a profundizar en próximas indagaciones, aunque se puede suponer como posible explicación que se deba al mismo proceso de distritalización y a las características propias de cada territorio sanitario.

Finalmente, quienes refieren confiar en las habilidades clínicas de los profesionales del otro nivel perciben que la atención suele ser más coordinada que quienes no experimentan dicha confianza.

\section{Conclusiónes}

La evaluación de la coordinación entre niveles es un punto fundamental para el funcionamiento de las RISS. Se considera que lo obtenido muestra rasgos comunes a otros países estudiados, con particularidades producto de la construcción de las redes de atención del nivel local. Los resultados de esta indagación, como está previsto en el marco del proyecto Equity LA II, constituyeron un insumo para el desarrollo de intervenciones con la participación de gestores, profesionales y equipo de investigación, que abordaron las problemáticas identificadas y tendiendo a la mejora de la coordinación.

\section{AGRADECIMIENTOS:}

Agradecemos a las personas entrevistadas que aceptaron compartir con los investigadores sus opiniones y conceptos; a las instituciones prestadoras de servicios de salud (hospitales y centros de salud) que han colaborado en el estudio; a la Comisión Europea, Programa FP7 cuyo apoyo financiero fue imprescindible para la realización del estudio.

\section{FINANCIAMIENTO:}

La investigación que ha conducido a estos resultados recibió fondos de la Unión Europea Séptimo Programa Marco (FP7/2007-2013) contrato número 305197, en el marco del proyecto "Impacto de las estrategias de integración de la atención en el desempeño de las redes de servicios de salud en diferentes sistemas de salud de América Latina (Equity LA II)". 


\section{Bibliografía}

1. Organización Panamericana de la Salud. Redes Integradas de Servicios de Salud. Conceptos, opciones de políticas y hoja de ruta para su implementación en las Américas. Washington: OPS/ OMS; 2010. [Disponible en https://www.paho.org/ uru/index.php?option $=$ com_docman\&view $=$ download\&alias $=145-$ redes-integradas-de-servicios-de-salud-aps-n4\&category_slug=publicaciones-sistemas-y-servicios-de-salud\&Itemid=307]. [Último acceso: agosto 2018].

2. Henao Martínez D, Vázquez Navarrete ML, Vargas Lorenzo I. Factores que influyen en la coordinación entre niveles asistenciales según la opinión de directivos y profesionales sanitarios. Gaceta Sanitaria.2009;23(4):280-286

3. Terraza Núñez R, Vargas Lorenzo I, Vázquez Navarrete ML. La coordinación entre niveles asistenciales: una sistematización de sus instrumentos y medidas. Gaceta Sanitaria. 2006; 20(6):485-95.

4. Berendsen AJ., Benneker WH., Schuling J, Rijkers-Koorn N, Slaets J.P, Meyboomde Jong B. Collaboration with general practitioners: preferences of medical specialists-a qualitative study. BMC. Health Services Research. 2006; 6, [155].

5. Andvig, E., Syse, J., Severinsson, E. Interprofessional collaboration in the mental health services in Norway. Nurs. Res. Pract. 2014; 849375.

6. Vargas I, Mogollón-Pérez A, De Paepe P, Ferreira da Silva MR, Unger JP, Vázquez ML. Do existing mechanisms contribute to improvements in care coordination across levels of care in health services networks? Opinions of the health personnel in Colombia and Brazil BMC Health Services Research 2015; 15:213

7. Vargas I, Mogollón-Pérez A, De Paepe P, Ferreira da Silva MR, Unger JP, Vázquez ML Barriers to healthcare coordination in marketbased and decentralized public health systems: a qualitative study in healthcare networks of Colombia and Brazil Health Policy and Planning. 2016; 31: 736-748

8. Vazquez ML, Vargas Ingrid, Garcia-Subirats I, Unger Jean-Pierre, De Paepe Pierre, Susana Mogollon-Perez A, et al. Doctors' experience of coordination across care levels and associated factors. A cross-sectional study in public healthcare networks of six Latin American countries. Social Science \& Medicine 2017; 182: 10-17

9. Munitis P. Sistema de referencia-contrarreferencia entre el segundo y el tercer nivel de salud en una red hospitalaria pediátrica en el conurbano bonaerense. Arch Argent Pediatr 2013; 111(5):405-411.

10. Mansilla C. Accesibilidad a las Interconsultas en el Centro de Especialidades Médicas Ambulatorias de Rosario de los pacientes adultos atendidos en el Primer Nivel de Atención. Buenos Aires: Becas de Investigación Ramón Carrillo- Arturo Oñativia; 2010. Anuario Comisión Nacional Salud Investiga. Ministerio de Salud. [Disponible en:http://www.msal.gov.ar/dis/wp-content/uploads/sites/11/2016/06/ Anuario_2010.pdf]. [Ultimo acceso: julio 2018]

11. Aronna A, Grande S, Herrmann J, Luppi I, Nestares G. Cómo se construyen y utilizan registros en atención primaria de la salud. En Miravet M, Arrona A, Grande S, Vilches N, Costaguta M, et al. Evaluación de tecnologías en Atención Primaria. Articulación Universidad- Servicios por el derecho a la salud. 1a ed. Rosario: Instituto de la Salud Juan Lazarte, 2011. p. 27-71

12. Orzuza G. Información en el primer nivel de atención. Revista Científica FCE. UNaM 2013; 17 (1) 95-113.

13. Vazquez ML, Vargas ML, Unger JP, De Paepe P, Mogollón-Perez A, Samico I et al. Evaluating the effectiveness of care integration strategies in different healthcare systems in Latin America: the EQUITY-LA II quasi-experimental study protocol. BMJ Open 2015; 5. [Disponible en: https://bmjopen.bmj.com/content/bmjopen/5/7/ e007037.full.pdf]. [Ultimo acceso: agosto 2018] 
14. Maceira D. Argentina: claves para coordinar un sistema de salud segmentado. Buenos Aires: CIPPEC; 2010. Documentos para políticas públicas. Recomendación No81.

15. Gobierno de Santa Fe. Santa Fe: Gobierno de Santa Fe, 2018 Red de salud integral y universal. [Disponible en: https://www.santafe.gov.ar/index.php/web/content/view/full/114560]. [Ultimo acceso: agosto 2018]

16. Municipalidad de Rosario. Secretaria de Salud Pública: Rosario, 2018 [Acceso 05 de febrero de 2018]. Centros de Salud y Hospitales municipales. Disponible en: https://www.rosario.gov.ar/web/servicios/ salud/hospitales/

17. Báscolo, E y N. Yavich. Gobernanza del desarrollo de la APS en Rosario, Argentina. Rev. salud pública. 2010; 12 (1): 89-104

18. Mansilla C; Urueta G; Monzon Valverde L, et al. Lo Real del trabajo. En la búsqueda de información sanitaria para la gestión. Investigación en Salud Publicación científica de la Secretaría de Salud Pública Municipal Vol. 10 - No 1 - Rosario, Enero- junio de 2013

19. Huerta A, Alonso M, Quadri I. La implementación del proceso de adscripción de ciudadanos al sistema de salud de Rosario y sus consecuencias en los procesos de trabajo en salud en Atención Primaria. Ponencia presentada en las VIII Jornadas Nacionales de Debate Interdisciplinario en Salud y Población. Instituto Gino Germani, Facultad de Ciencias Sociales, Universidad de Buenos Aires. Agosto de 2009.

20. Dirección General de Estadística Municipalidad de Rosario. Anuario de Población y Estadísticas Vitales $2^{\circ} 15$ Ciudad de Rosario. Rosario: Municipalidad de Rosario, 2015

21. Øvretveit, J. Does Clinical Coordination Improve Quality and Save Money? London: Health Foundation; 2011

22. Rovere, M. 2006. Redes en salud; los grupos, las instituciones, la comunidad. Ed. El ágora, Buenos Aires, Argentina.

23. Quadri, A. Accesibilidad a estudios de Resonancia Magnética Nuclear en pacientes atendidos en consultorios externos del Centro de Especialidades Médicas Ambulatorias de Rosario en el período comprendido entre julio de 2011 y junio de 2012. Inv en salud. 2015, 11 (1): $21-43$. 